APPEARED IN BULLETIN OF THE

AMERICAN MATHEMATICAL SOCIETY

Volume 26, Number 2, April 1992, Pages 264-268

\title{
A THEORY OF ALGEBRAIC COCYCLES
}

\author{
Eric M. Friedlander and H. Blaine Lawson, Jr.
}

\begin{abstract}
We introduce the notion of an algebraic cocycle as the algebraic analogue of a map to an Eilenberg-MacLane space. Using these cocycles we develop a "cohomology theory" for complex algebraic varieties. The theory is bigraded, functorial, and admits Gysin maps. It carries a natural cup product and a pairing to $L$-homology. Chern classes of algebraic bundles are defined in the theory. There is a natural transformation to (singular) integral cohomology theory that preserves cup products. Computations in special cases are carried out. On a smooth variety it is proved that there are algebraic cocycles in each algebraic rational $(p, p)$-cohomology class.
\end{abstract}

In this announcement we present the outlines of a cohomology theory for algebraic varieties based on a new concept of an algebraic cocycle. Details will appear in [FL]. Our cohomology is a companion to the $L$-homology theory recently studied in $[\mathrm{L}, \mathrm{F}, \mathrm{L}-\mathrm{F} 1, \mathrm{~L}-\mathrm{F} 2, \mathrm{FM}]$. This homology is a bigraded theory based directly on the structure of the space of algebraic cycles. It admits a natural transformation to integral homology that generalizes the usual map taking a cycle to its homology class. Our new cohomology theory is similarly bigraded and based on the structure of the space of algebraic cocycles. It carries a ring structure coming from the complex join (an elementary construction of projective geometry), and it admits a natural transformation $\Phi$ to integral cohomology. Chern classes are defined in the theory and transform under $\Phi$ to the usual ones. Our definition of cohomology is very far from a duality construction on $L$-homology. Nonetheless, there is a natural and geometrically defined Kronecker pairing between our "morphic cohomology" and $L$-homology.

The foundation stone of our theory is the notion of an effective algebraic cocycle, which is of some independent interest. Roughly speaking, such a cocycle on a variety $X$, with values in a projective variety $Y$, is a morphism from $X$ to the space of cycles on $Y$. When $X$ is normal, this is equivalent (by "graphing") to a cycle on $X \times Y$ with equidimensional fibres over $X$. Such cocycles abound in algebraic geometry and arise naturally in many circumstances. The simplest perhaps is that of a flat morphism $f: X \rightarrow Y$ whose corresponding cocycle associates to $x \in X$, the pullback cycle $f^{-1}(\{x\})$. Many more arise naturally from synthetic constructions in projective geometry. We show that every variety is rich in cocycles. Indeed if

1991 Mathematics Subject Classification. Primary 14F99, 14 C05.

Key words and phrases. Algebraic cycle, Chow variety, algebraic cocycle, cohomology.

Received by the editors April 4, 1991

The first author was partially supported by the IHES, NSF Grant DMS 8800657, and NSA Grant MDA904-90-H-4006. The second author was partially supported by NSF DMS 8602645 
$X$ is smooth and projective, then every rational cohomology class that is Poincare dual to an algebraic cycle is represented by (i.e., is $\Phi \otimes \mathbb{Q}$ of) an algebraic cocycle.

In what follows the word variety will denote a reduced, irreducible, locally closed subscheme of some complex projective space. Such a variety is called projective if it is in fact closed (equivalently, compact) in some projective space.

Definition 1. Given a projective variety $Y \subset P^{n}$ with a fixed embedding, we denote by $C_{d}^{s}(Y)$ the algebraic set (the "Chow set") of effective cycles of codimension- $s$ and degree $d$ with support in $Y$. For an arbitrary variety $X$ we then define an effective algebraic cocycle on $X$ with values in $Y$ to be a continuous algebraic map $\varphi: X \rightarrow C_{d}^{s}(Y)$ (i.e., a morphism $\varphi: \tilde{X} \rightarrow C_{d}^{s}(Y)$ from the weak normalization $\tilde{X}$ of $X)$. The space of all such cocycles provided with the compact-open topology will be denoted by $C_{d}^{s}(X ; Y)$.

Note that $C_{d}^{s}(X ; Y)$ is a priori a hybrid construction consisting of algebrogeometric objects but carrying the compact-open topology. However, for normal projective varieties $X$, it has a purely algebraic description.

Proposition 2. If $X$ is projective and normal, then the space $C_{d}^{s}(X ; Y)$ admits the structure of a locally closed, reduced subscheme of some complex projective space.

Formal direct sum determines an abelian topological monoid structure on the spaces

$$
C^{s}(X ; Y) \stackrel{\text { def }}{=} \coprod_{d \geq 0} C_{d}^{s}(X ; Y) .
$$

This structure is proved to be independent of the projective embedding chosen for $Y$. In analogy with the construction of $L$-homology, we have the following definition.

Definition 3. Let $X$ and $Y$ be varieties, with $Y$ projective. Denote by $Z^{s}(X ; Y)$ the homotopy theoretic group completion of $C^{s}(X ; Y)$ (i.e., the loops on the classifying space of $\left.C^{s}(X ; Y)\right)$,

$$
Z^{s}(X ; Y) \equiv \Omega B\left(C^{s}(X ; Y)\right) .
$$

Then the bivariant morphic cohomology of $X$ with coefficients in $Y$ is defined to be the homotopy groups of $Z^{s}(X ; Y)$,

$$
L^{s} H^{q}(X ; Y) \equiv \pi_{2 s-q}\left(Z^{s}(X ; Y)\right), \quad 2 s \geq q \geq 0 .
$$

The first fundamental result concerning these spaces is the Algebraic Suspension Theorem, which asserts that the algebraic suspension maps $C_{d}^{s}(X ; Y) \rightarrow$ $C_{d}^{s}(X ; \not \Varangle Y)$, introduced in [L], induce a homotopy equivalence

$$
Z^{s}(X ; Y) \stackrel{\cong}{\longrightarrow} Z^{s}(X ; \not \nless Y)
$$

and thus an isomorphism

$$
L^{s} H^{q}(X ; Y) \cong L^{s} H^{q}(X ; \not \not Y)
$$

for all $s$ and $q$. 
Although this theory has been developed in the "bivariant context" of Definition 3 , we shall focus our attention here on the important special case in which $Y$ is some projective space $P^{N}$. We know from [L] that $\Omega B\left(C^{s}\left(P^{N}\right)\right)$ has the homotopy type of a generalized Eilenberg-MacLane space

$$
\Omega B\left(C^{s}\left(P^{N}\right)\right) \cong K(\mathbf{Z}, 0) \times K(\mathbf{Z}, 2) \times \cdots \times K(\mathbf{Z}, 2 s), \quad N \geq s .
$$

In particular, this homotopy type is independent of $N \geq s$. We conclude that $\Omega B\left(C^{s}\left(P^{N}\right)\right)$ "modulo" $\Omega B\left(C^{s-1}\left(P^{N-1}\right)\right)$ represents cohomology. This motivates the following

Definition 4. For any variety $X$ and any $s \geq 0$, let $Z^{s}(X)$ denote the homotopy fibre of the natural map $B C^{s-1}\left(X, P^{s-1}\right) \rightarrow B C^{s}\left(X, P^{s}\right)$,

$$
Z^{s}(X) \equiv \operatorname{htyfib}\left\{B C^{s-1}\left(X, P^{s-1}\right) \rightarrow B C^{s}\left(X, P^{s}\right)\right\} .
$$

For any $0 \leq q \leq 2 s$, the morphic cohomology group $L^{s} H^{q}(X)$ is defined by

$$
L^{s} H^{q}(X) \equiv \pi_{2 s-q}\left(Z^{s}(X)\right) .
$$

Definitions 3 and 4 are related as follows.

Theorem 5. For any variety $X$ and any $N \geq s \geq 0$, there is a natural homotopy equivalence

$$
Z^{s}\left(X ; P^{N}\right) \cong Z^{0}(X) \times Z^{1}(X) \times \cdots \times Z^{s}(X) .
$$

The splitting asserted in Theorem 5 arises from natural maps

$$
S P^{\infty}\left(P^{N}\right) \rightarrow S P^{\infty}\left(P^{k}\right), \quad k \leq N
$$

obtained by viewing $P^{N}$ as $S P^{N}\left(P^{1}\right)$ and $P^{k}$ as $S P^{k}\left(P^{1}\right)$, where $S P^{j}\left(P^{n}\right)$ denotes the $\mathrm{j}$-fold symmetric product of $P^{n}$.

We view morphic cohomology as the theory corresponding to "algebraic" as opposed to "arbitrary continuous" maps from $X$ into Eilenberg-MacLane spaces. This perspective is formalized in the following.

Theorem 6. For any variety $X$, the elementary complex join operation induces a natural ring structure on

$$
L^{\cdot} H^{\cdot}(X) \equiv \bigoplus_{s \geq 0} L^{s} H^{\cdot}(X)
$$

Furthermore, there is a natural transformation of graded rings

$$
\Phi: L^{\cdot} H^{\cdot}(X) \rightarrow H^{\cdot}(X ; \mathbf{Z}),
$$

and, if $X$ is projective then

$$
\Phi\left(L^{s} H^{2 s-j}(X)\right) \otimes \mathbf{C} \subset H^{s, s-j} \oplus H^{s-1, s-j+1} \oplus \cdots \oplus H^{s-j, j},
$$

where $H^{p, q}$ denotes the $(p, q)$ th Dolbeault component of $H^{p+q}(X ; \mathbf{C})$.

The existence of a ring structure in morphic cohomology provides it with a structure not possessed by $L$-homology. On the other hand, the natural operations on $L$-homology constructed in $[\mathrm{FM}]$ via the join operation naturally determine operations on our morphic cohomology groups.

The restriction on the image of $\Phi$ given in Theorem 6 is complemented by the following existence result. A key ingredient in its proof is the total Chern class map of $[\mathrm{LM}]$,

$$
B U_{s} \rightarrow C^{s}\left(P^{\infty}\right)
$$

which can be viewed geometrically as the inclusion of degree 1 cycles into the space of all cycles on $P^{N}$ for $\mathrm{N}$ sufficiently large. 
Theorem 7. Let $E$ be a vector bundle over $X$ generated by its global sections, over a variety $X$. Then there are naturally defined chern classes

$$
c_{k}(E) \in L^{k} H^{2 k}(X)
$$

with the property that $\Phi\left(c_{k}(E)\right) \in H^{2 k}(X ; \mathbf{Z})$ is the usual kth chern class of $E$. Consequently, if $X$ is a smooth projective variety, then the Poincare dual of the fundamental class of each algebraic subvariety lies in the subring of $H^{*}(X ; \mathbf{Z})$ generated $\Phi(L \cdot H \cdot(X))$.

Not surprisingly, codimension- 1 morphic cohomology is the easiest to compute. We have the following computation.

Theorem 8. Let $X$ be a projective variety. Then

$$
L^{1} H^{q}(X)= \begin{cases}\mathbf{Z} & \text { if } q=0, \\ H^{1}(X ; \mathbf{Z}) & \text { if } q=1, \\ H^{1,1}(X ; \mathbf{Z}) & \text { if } q=2, \\ 0 & \text { otherwise }\end{cases}
$$

where $H^{1,1}(X ; \mathbf{Z})$ denotes $H^{2}(X ; \mathbf{Z}) \cap \rho^{-1} H^{1,1}(X ; \mathbf{C})$ and where $\rho: H^{2}(X ; \mathbf{Z}) \rightarrow$ $H^{2}(X ; \mathbf{C})$ is the coefficient homomorphism.

In $[\mathrm{F}]$ a result similar to Theorem 8 but only applying to smooth projective varieties was proved for $L$-homology. This suggests that morphic cohomology and $L$-homology should satsify some form of duality. One possible candidate for a possible duality pairing is given in the following proposition.

Proposition 9. For any variety $X$, there is a natural Kronecker pairing between L-homology and morphic cohomology,

$$
L^{s} H^{q}(X) \otimes L_{r} H_{q}(X) \rightarrow \mathbf{Z},
$$

which is naturally compatible with the usual Kronecker pairing

$$
H^{q}(X ; \mathbf{Z}) \otimes H_{q}(X ; \mathbf{Z}) \rightarrow \mathbf{Z} .
$$

The definition of this pairing is pleasingly geometric. Namely, given a cycle $W$ on $X \times P^{N}$ and a cycle $Z$ on $\mathrm{X}$, we take the image in $H_{*}\left(P^{N} ; \mathbf{Z}\right)$ of the fundamental class of the restriction of $W$ to $Z$.

\section{REFERENCES}

[F] E. Friedlander, Algebraic cycles, Chow varieties, and Lawson homology, Compositio Math. 77 (1991), 55-93.

[FL] E. Friedlander and H. B. Lawson, A theory of algebraic cocycles, Ann. of Math. (to appear). $[\mathrm{FM}]$ E. Friedlander and B. Mazur, Filtrations on the homology of algebraic varieties (to appear).

[L-F1] P. Lima-Filho, On a homology theory for algebraic varieties, IAS preprint, 1990.

[L-F2] Completions and fibrations for topological monoids and excision for Lawson homology, Compositio Math., (1991).

[L] H. B. Lawson, Jr., Algebraic cycles and homotopy theory, Ann. of Math. (2) 129 (1989), 253-291.

[LM] H. B. Lawson, Jr. and M.-L. Michelsohn, Algebraic cycles, Bott periodicity, and the Chern characteristic map, The Mathematical Heritage of Herman Weyl, Amer. Math. Soc., Providence, RI, 1988, pp. 241-264.

Department of Mathematics, Northwestern University, Evanston, Illinois 60208

Department of Mathematics, State University of New York, Stony Brook, New YORK 11794 